Susanne Tietz-Weber

Interessengruppen und Rechnungslegungsregeln 
GABLER EDITION WISSENSCHAFT 
Susanne Tietz-Weber

\section{Interessengruppen und Rechnungslegungsregeln}

Eine Analyse des Umsetzungsprozesses

der 4. EG-Richtlinie in das

Bilanzrichtlinien-Gesetz

Mit einem Geleitwort von Prof. Dr. Günther Gebhardt

Deutscher Universitäts-Verlag 
Bibliografische Information Der Deutschen Bibliothek

Die Deutsche Bibliothek verzeichnet diese Publikation in der Deutschen Nationalbibliografie; detaillierte bibliografische Daten sind im Internet über <http://dnb.ddb.de> abrufbar.

Dissertation Universität Frankfurt/Main, 2005

\section{Auflage März 2006}

Alle Rechte vorbehalten

(C) Deutscher Universitäts-Verlag I GWW Fachverlage GmbH, Wiesbaden 2006

Lektorat: Ute Wrasmann / Stefanie Brich

Der Deutsche Universitäts-Verlag ist ein Unternehmen von Springer Science+Business Media. www.duv.de

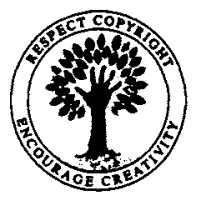

Das Werk einschließlich aller seiner Teile ist urheberrechtlich geschützt. Jede Verwertung außerhalb der engen Grenzen des Urheberrechtsgesetzes ist ohne Zustimmung des Verlags unzulässig und strafbar. Das gilt insbesondere für Vervielfältigungen, Ubbersetzungen, Mikroverfilmungen und die Einspeicherung und Verarbeitung in elektronischen Systemen.

Die Wiedergabe von Gebrauchsnamen, Handelsnamen, Warenbezeichnungen usw. in diesem Werk berechtigt auch ohne besondere Kennzeichnung nicht zu der Annahme, dass solche Namen im Sinne der Warenzeichen- und Markenschutz-Gesetzgebung als frei zu betrachten wären und daher von jedermann benutzt werden dürften.

Umschlaggestaltung: Regine Zimmer, Dipl.-Designerin, Frankfurt/Main

Druck und Buchbinder: Rosch-Buch, ScheBlitz

Gedruckt auf säurefreiem und chlorfrei gebleichtem Papier

Printed in Germany

ISBN 3-8350-0242-2 


\section{Geleitwort}

Rechnungslegungsregeln nehmen Einfluss auf die Verteilung von Informationen und von Überschüssen bzw. Vermögen. Die Festlegung von Rechnungslegungsregeln greift in Verteilungsprozesse ein und ist damit von eminenter Bedeutung für die betroffenen Personengruppen und Institutionen, die ihre Interessen in den Prozessen zur Änderung von Rechnungslegungsregeln einbringen werden. Die Untersuchung dieser Prozesse liefert daher wichtige Erkenntnisse zum Verstänḍis der Entwicklung von Rechnungslegungsregeln.

Hier setzt die Arbeit von Frau Tietz-Weber an, die den Einfluss von Interessengruppen auf ein konkretes wichtiges Regulierungsprojekt - die Umsetzung der 4. EG-Richtlinie in deutsches Bilanzrecht - umfassend analysiert und qualifiziert. Dabei legt die theoretisch fundierte Darstellung von politischen Entscheidungsprozessen im Allgemeinen und im Bereich der Rechnungslegung ein wichtiges Fundament für die empirische Analyse. Diese liefert durch die Auswertung von mehr als 1.000 verbalen Äußerungen von vier Interessengruppen in Zeitschriften und Protokollen sowie der unterschiedlichen Gesetzentwürfe zahlreiche interessante Erkenntnisse über das Zustandekommen von Rechnungslegungsregeln. Die Äußerungen der Interessengruppen zu einzelnen Problemen wurden verdichtet, inhaltlich analysiert und kodiert, so dass relevante Fragestellungen statistisch untersucht werden konnten. Beispielhaft seien hier nur die Koalitionsanalysen, die unterschiedlichen Erfolgsanalysen und die Analyse des Regulierungsprozesses im Zeitablauf herausgegriffen.

Initiiert wurde diese Arbeit noch von meinem leider zu früh verstorbenen Frankfurter Kollegen Dieter Ordelheide, der zu Recht nicht nur im deutschen Sprachraum als ein (wenn nicht der) Pionier auf dem Gebiet der politischen Ökonomie der Rechnungslegung gilt.

Mit der vorliegenden Arbeit liefert Frau Tietz-Weber einen wichtigen Baustein im Bereich der Forschung zur politischen Ökonomie der Rechnungslegung. Der Umsetzungsprozess der 4. EG-Richtlinie in deutsches Recht wird sehr differenziert ausgewertet und dabei ein methodisches Instrumentarium (weiter-) entwickelt, das sich zur Analyse von Regulierungsprozessen nicht nur im Bereich der Rechnungslegung sehr gut eignet. Ich wünsche dieser gelungenen und in ihren Ergebnissen höchst interessanten Arbeit eine positive Aufnahme in Theorie und Praxis. 


\section{Vorwort}

Die vorliegende Arbeit wurde vom Fachbereich Wirtschaftswissenschaften der Johann Wolfgang Goethe-Universität, Frankfurt am Main, im September 2005 als Dissertation angenommen. Sie entstand während meiner Zeit als wissenschaftliche Mitarbeiterin an der Professur für Internationales Rechnungswesen und als externe Doktorandin und wissenschaftliche Mitarbeiterin an der Professur für Wirtschaftsprüfung.

Mein erster und besonderer Dank gilt meinem viel zu früh verstorbenen akademischen Lehrer und Doktorvater, Herrn Prof. Dr. Dr. h.c. Dieter Ordelheide, der mein Promotionsvorhaben unterstützte und mit anregenden Diskussionen begleitete. Durch sein Interesse und die Neugierde an der Untersuchung des Entwicklungsprozesses von Rechnungslegungsregeln wurde auch in mir die Leidenschaft für dieses Thema geweckt. Aber nicht nur in fachlicher, auch in menschlicher Hinsicht hat er mich stark geprägt und zwar deutlich über die Freude an gutem Rotwein hinaus. Herrn Professor Dr. Günther Gebhardt danke ich, dass er nach dem Tod von Herrn Professor Ordelheide ohne Zögern die weitere Betreuung meiner Arbeit übernommen hat und im weiteren Verlauf die Fertigstellung nicht nur durch zahlreiche Anmerkungen und Diskussionen stets gefördert hat. Mein Dank gilt auch Professor Dr. Hans-Joachim Böcking für die zügige Erstellung des Zweitgutachtens, Herrn Professor Dr. Winfried Mellwig für den Vorsitz der Prüfungskommission und Herrn Professor Dr. Uwe Hassler für die Beteiligung als Prüfer und Anregungen für den statistischen Teil der Arbeit.

Der Graduiertenförderung des Landes Hessen danke ich für die großzügige Unterstützung durch die Gewährung eines Stipendiums und der Deutschen Forschungsgemeinschaft für die Finanzierung meiner Stelle während meiner Tätigkeit am Lehrstuhl für Internationales Rechnungswesen. Bei meinen Kollegen von beiden Lehrstühlen möchte ich für die schöne gemeinsame Zeit bedanken. Meiner Familie möchte ich an dieser Stelle für ihre andauernde Unterstützung danken: Meine Schwester PD Dr. Christiane Tietz unterstützte mich durch ihr großes Engagement bei der Korrektur meiner Arbeit und ihre unermüdliche Diskussionsbereitschaft. Mein Vater Professor Dr. Reinhard Tietz war mir durch die zahlreichen wertvollen Anmerkungen und seine ständige Gesprächsbereitschaft ein große Hilfe. Meine Mutter Ursula Tietz hat für ihren Einsatz beim Korrekturlesen und für die kulinarische Unterstützung einen besonderen Dank verdient.

Ganz besonders herzlich möchte ich meinem Mann Dr. Christoph Weber danken für seine andauernde Diskussionsbereitschaft, das Korrekturlesen und die moralische Unterstützung. $\mathrm{Er}$ stellte in dieser Zeit seine Interessen oft zurück, damit ich mich meinen Interessengruppen widmen konnte. 


\section{Inhaltsverzeichnis}

Abbildungsverzeichnis.......................................................................................................................XV

Tabellenverzeichnis .............................................................................................................XVII

Verzeichnis der verwendeten Symbole ................................................................................. XXI

Abkürzungsverzeichnis ........................................................................................................ XXIII

Teil I: Einleitung ....................................................................................................................

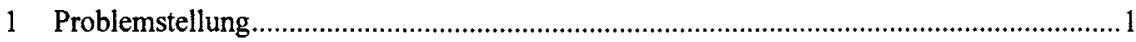

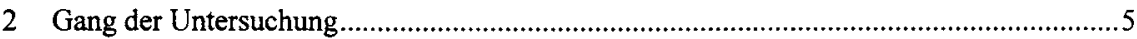

Teil II: Der Einfluss von Interessengruppen auf den politischen Entscheidungsprozess ................................................................................................9

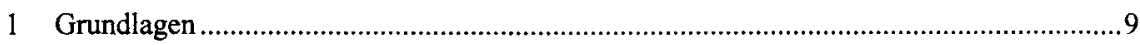

1.1 Terminologische Einordnung des Begriffs „Interessengruppe“ ...............................9

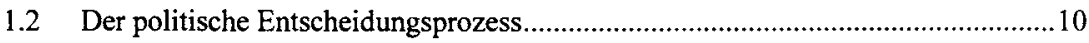

1.3 Das Verhalten der Akteure aus ökonomischer Sicht .............................................12

2 Die Theorie der Regulierung......................................................................................13

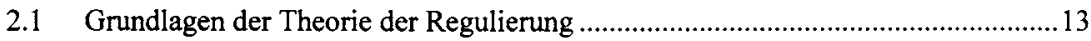

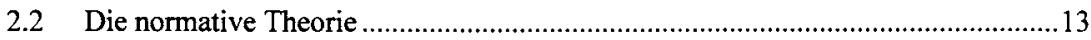

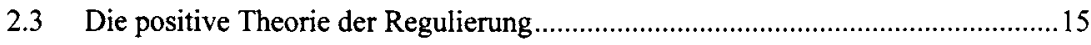

3 Die Akteure im politischen Prozess ......................................................................... 18

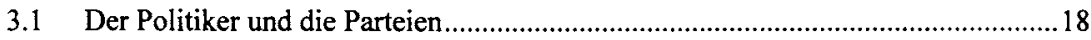

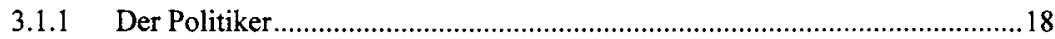

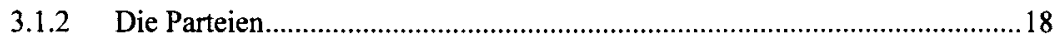

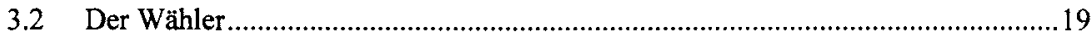

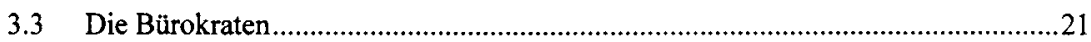

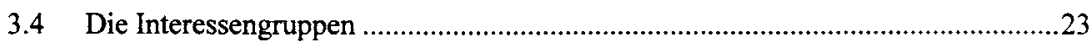

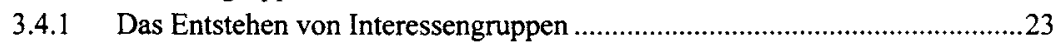

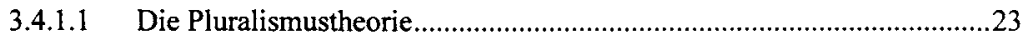

3.4.1.2 Die Logik kollektiven Handelns ..........................................................24

3.4.2 Das Einflusspotenzial von Interessengruppen ...............................................27

3.4.2.1 Ursachen des Einflusses von Interessengruppen ......................................27

3.4.2.2 Die Durchsetzungskraft der Interessengruppe beeinflussende

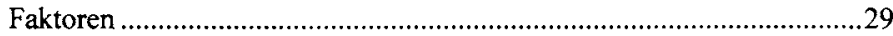

3.4.2.2.1 Gruppeninterne Faktoren des Einflusses ........................................29

3.4.2.2.2 Äußere Faktoren des Einflusses......................................................30

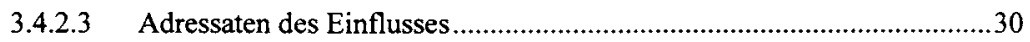

3.4.2.3.1 Kriterien für die Wahl des geeigneten Adressaten ...........................30 


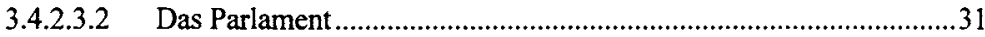

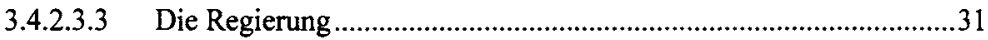

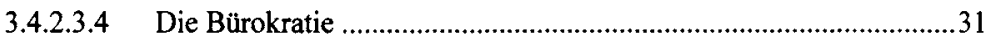

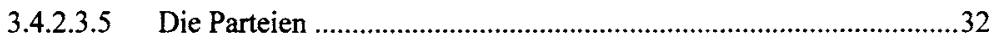

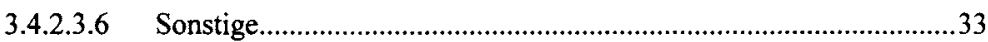

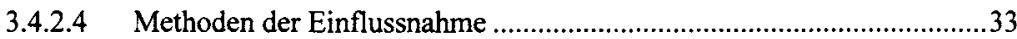

3.4.3 Der Einfluss von Interessengruppen unter Wettbewerbsbedingungen ............34

3.4.4 Studien zur Rolle der Interessengruppen im politischen Entscheidungsprozess

Teil III: Der Einfluss von Interessengruppen auf die Entwicklung von Rechnungslegungsregeln .

1 Die Theorie der Regulierung im Bereich der Rechnungslegung ………........................41

1.1 Begriff und Zwecke der Rechnungslegung .......................................................4 41

1.2 Die Regulierung der Rechnungslegung ..............................................................4

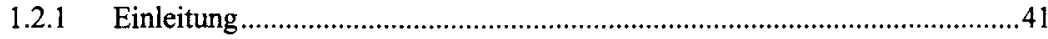

1.2.2 Normative Ansätze der Theorie der Regulierung der Rechnungslegung .......42

1.2.3 Positive Ansätze der Theorie der Regulierung der Rechnungslegung ............45

1.2.3.1 Grundidee der Positive-accounting-theory .................................................45

1.2.3.2 Krisentheorie und Regulierung der Rechnungslegung .............................46

1.2.4 Andere Erklärungsansätze ......................................................................47

2 Der Einfluss von Interessengruppen auf den Normsetzungsprozess im Bereich der

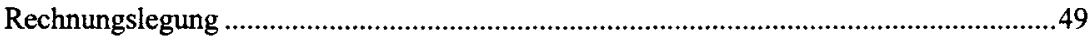

2.1 Die handelnden Akteure ..................................................................................49

2.2 Theoretische Überlegungen zur Einflussnahme auf den

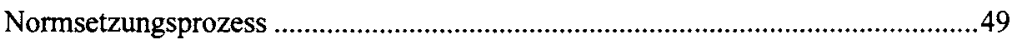

2.2.1 Der politische Entscheidungsprozess im Bereich der Rechnungslegung .......49

2.2.2 Überlegungen im Rahmen der Positive-accounting-theory ..............................51

2.2.2.1 Theoretische Ansätze ..............................................................................51

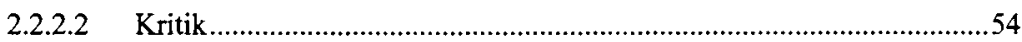

2.2.3 Das ökonomisch geprägte Modell von Sutton................................................56

2.2.4 Weitere Überlegungen zur Einflussnahme auf den
Normsetzungsprozess ....................................................................................58

3 Studien zur Rolle der Interessengruppen bei der Entwicklung von

Rechnungslegungsregeln.........................................................................................5

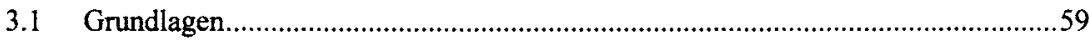

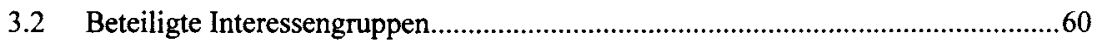

3.3 Gründe für die Beteiligung .............................................................................62

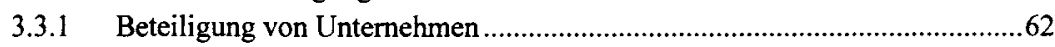

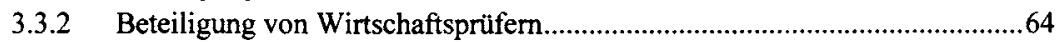

3.3.3 Beteiligung von Hochschullehrern ..............................................................65 
3.4 Inhaltliche Ausrichtungen und Wege der Einflussnahme..................................66

3.5 Erfolgreiche Einflussnahme der Interessengruppen ....................................67

Teil IV: Der Einfluss von Interessengruppen auf die Umsetzung der 4. EGRichtlinie in das Bilanzrichtlinien-Gesetz..........................................................71

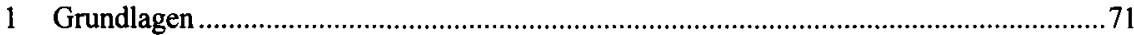

1.1 Die Regulierung der Rechnungslegung in Deutschland ...................................71

1.2 Die Aufgaben der Rechnungslegung ............................................................. 72

1.3 Der Gesetzgebungsprozess in der Bundesrepublik Deutschland.............................73

1.4 Studien zur Rolle der Interessengruppen im politischen Prozess in

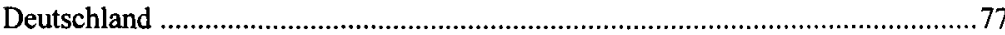

2 Empirische Auswertung des Einflusses von Interessengruppen auf den Umsetzungsprozess der 4. EG-Richtlinie............................................................8

2.1 Der Untersuchungsgegenstand - Die Umsetzung der 4. EG-Richtlinie in das Bilanzrichtlinien-Gesetz ..............................................................8

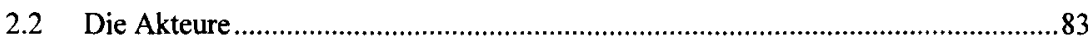

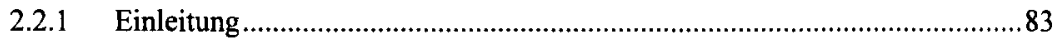

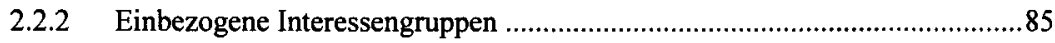

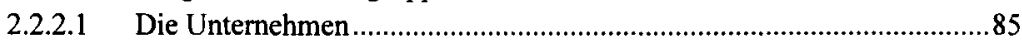

2.2.2.1.1 Die Spitzenverbånde der deutschen Wirtschaft ..............................85

2.2.2.1.2 Der Arbeitskreis, ,Rechnungslegungsvorschriften in der EGKommission" der Gesellschaft für Finanzwirtschaft in der Unternehmensführung e.V........................................................85

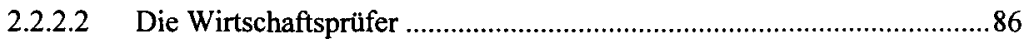

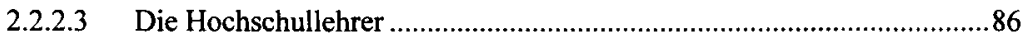

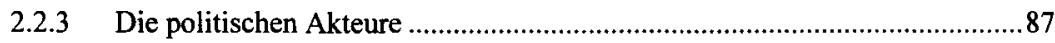

2.3 Zeittafel der Vorstufen des Bilanzrichtlinien-Gesetzes und der verschiedenen öffentlichen Stellungnahmen der betrachteten

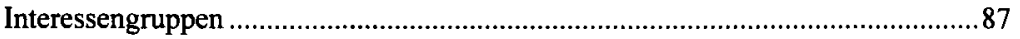

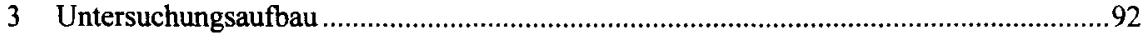

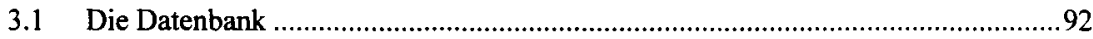

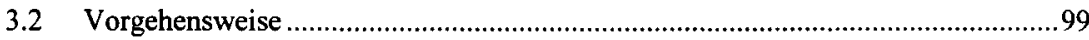

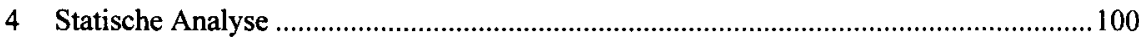

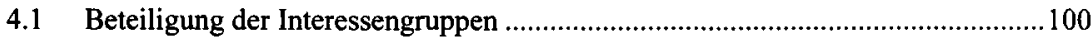

4.1.1 Aktivität der untersuchten Interessengruppen ..................................... 100

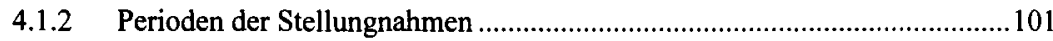

4.1.3 Inhaltliche Schwerpunkte der Stellungnahmen ......................................... 102

4.1.4 Inhaltliche Zielrichtung der Stellungnahmen ......................................... 107

4.2 Statische Erfolgsanalyse der Interessengruppen ............................................... 121

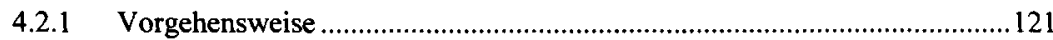

4.2.2 Erfolg der Interessengruppen ................................................... 123 


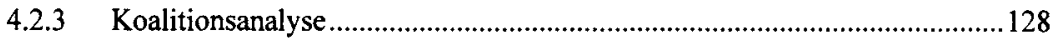

4.2.4 Zusammensetzung und Erfolg der unterschiedlichen Koalitionen ...............132

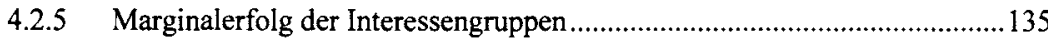

4.2.6 Ähnlichkeiten zwischen den Interessengruppen .........................................138

4.2.7 Distanzsummen und Koalitionsstruktur...................................................... 140

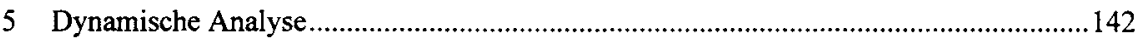

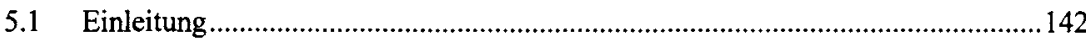

5.2 Der Umsetzungsprozess dargestellt am Beispiel des Geschäfts- oder Firmenwerts

5.2.1 Klassifizierung des Geschäfts- oder Firmenwertes als

Vermögensgegenstand oder Bilanzierungshilfe .........................................142

5.2.2 Aktivierungswahlrecht oder Aktivierungspflicht ......................................146

5.2.3 Folgebewertung des Geschäfts- oder Firmenwerts........................................ 147

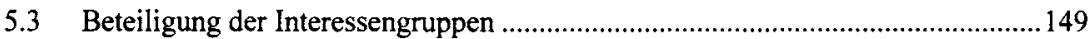

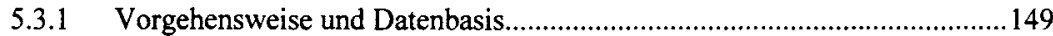

5.3.2 Ausmaß und Zeitpunkt der Beteiligung der Interessengruppen ....................150

5.3.2.1 Intensität der Aktivität der Interessengruppen.........................................150

5.3.2.2 Periode der Stellungnahmen ............................................................... 151

5.4 Veränderungen durch die Betrachtung des Prozesses ............................................152

5.4.1 Meinungsänderungen der Interessengruppen im Zeitablauf..........................152

5.4.2 Statischer Erfolg der Interessengruppen nach Meinungsänderung................153

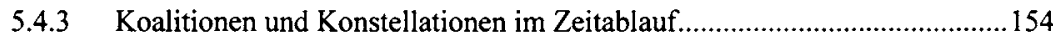

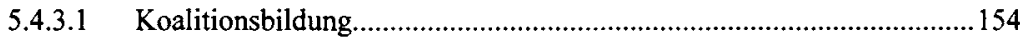

5.4.3.2 Veränderung der Koalitionsstruktur im Zeitablauf..................................156

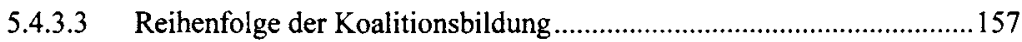

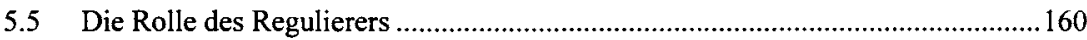

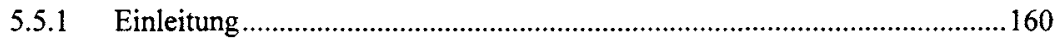

5.5.2 Anzahl der behandelten Rechnungslegungsprobleme ................................. 161

5.5.3 Interessengruppenkonstellationen - Meinung des Regulierers ...................... 161

5.5.4 Meinungswechsel des Regulierers ............................................................ 163

5.6 Erfolgsanalyse der Interessengruppen im Zeitablauf.........................................167

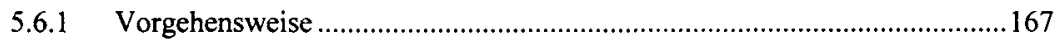

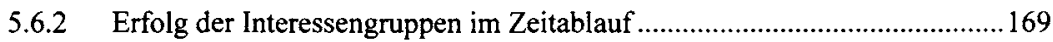

5.6.3 Koalitionsanalyse - Vergleich Regierungsentwurf $1982 \mathrm{mit}$

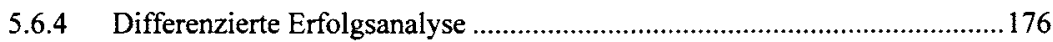

5.7 Regressionen zu Einflussfaktoren des Erfolges der Interessengruppen ................179

6 Langfristige Erfolgsanalyse......................................................................................... 182

Teil V: Zusammenfassung...................................................................................................187 


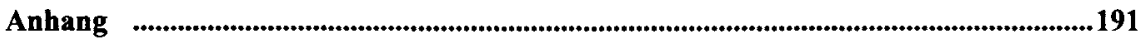

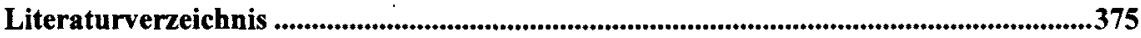

Verzeichnis der öffentlichen Stellungnahmen........................................................400

Verzeichnis der Gesetze, EU-Richtlinien, Gesetzentwürfe, sonstigen Materialien

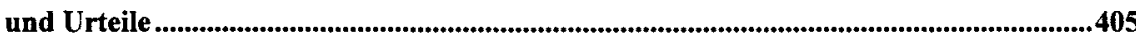




\section{Abbildungsverzeichnis}

Abb. 1: Adressaten und Methoden der Einflussnahme der Interessengruppen 34

Abb. 2: Verteilung der Aktivitäten auf Bereiche

Abb. 3: Erfolgszuwachs durch Koalitionspartner 136

Abb. 4: Marginaler Erfolgsbeitrag durch Koalitionsbeitritt .............................................138

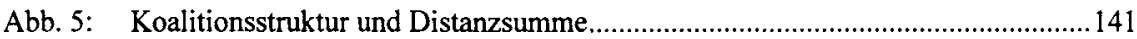

Abb. 6: Aktivität und Erfolg der KRW im Zeitablauf..................................................170

Abb. 7: Aktivität und Erfolg des IDW im Zeitablauf ................................................. 170

Abb. 8: Aktivität und Erfolg der GEFIU im Zeitablauf .................................................171

Abb. 9: Aktivität und Erfolg der SpVdW im Zeitablauf ..................................................171

Abb. 10: Distanz fortwirkender Stellungnahmen zum Regulierer im Zeitablauf..................172 


\section{Tabellenverzeichnis}

Tab. 1: Beteiligte Interessengruppen am Umsetzungsprozess der 4. EGRichtlinie .84

Tab. 2: Stellungnahmen der KRW, IDW, GEFIU und SPVDW zur Umsetzung

4. EG-Richtlinie und zu den Entwürfen eines Bilanzrichtlinien-Gesetzes

Tab. 3: Gliederung der Rechnungslegungsprobleme.

Tab. 4: Aufnahme der Stellungnahme, Zerlegung in einzelne Aussagen und Zuordnung einer Gliederungsnummer in der Textdatenbank

Tab. 5: Bündelung der Stellungnahmen, Definition der

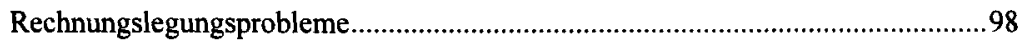

Tab. 6: Problemaktivität der Interessengruppen ..............................................................100

Tab. 7: Perioden der Erststellungnahmen........................................................................ 101

Tab. 8: Übersicht über die Anzahl der Rechnungslegungsprobleme, der zu ihnen verfassten Stellungnahmen, die Problemaktivität und den Anteil der Stellungnahmen.

Tab. 9: Häufigkeit der Kriterien der Zielrichtung .........................................................107

Tab. 10: Häufigste Zielrichtungen der Stellungnahmen für die Bereiche 01.-04.

Tab. 11: Auszug aus Anhang A-3: Liste aller Rechnungslegungsprobleme .........................123

Tab. 12: Kodierung der Stellungnahmen.......................................................................123

Tab. 13: Auszug aus Anhang A-5: Datenbasis für die Statische Analyse.............................123

Tab. 14: Erfolg der Erststellungnahmen der Interessengruppen ...........................................124

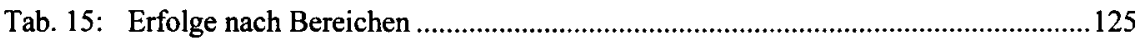

Tab. 16: Erfolge abhängig von der vertretenen Zielrichtung .............................................126

Tab. 17: Erfolg der Interessengruppen bei Äußerung von mindestens zwei

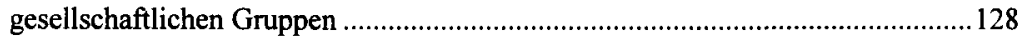

Tab. 18: Anzahl und Erfolg der Koalitionen ohne Gegenstimmen .................................... 129

Tab. 19: Anzahl und Erfolg der Koalitionen mit Gegenstimmen.......................................130

Tab. 20: Erfolg der Interessengruppen in den Koalitionen ohne Gegenstimmen..................130

Tab. 21: Anzahl und Erfolg der Interessengruppen in Koalitionen mit Gegenstimmen

Tab. 22: Anzahl und Erfolg der Interessengruppen alleine gegen andere Interessengruppen.

Tab. 23: Zusammenfassung der Erfolgsquote der Interessengruppen mit und ohne Gegenstimmen

Tab. 24: Zusammensetzung, Anzahl und Erfolg der unterschiedlichen

Konstellationen

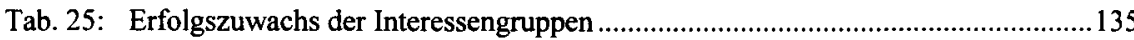

Tab. 26: Marginaler Erfolgsbeitrag der Interessengruppen ........................................... 137

Tab. 27: Anzahl und Erfolg der mit einer bestimmten Interessengruppe übereinstimmend vertretenen Meinung und Übereinstimmungsquote 
Tab. 28: Aktivitätsverwandschaft und Korrelationskoeffizient für die Aktivitäten der Interessengruppen.

Tab. 29: Ermittlung der Distanzsumme ........................................................................... 140

Tab. 30: Distanzsummen der Konstellationen.................................................................... 140

Tab. 31: Anzahl der zu einem Rechnungslegungsproblem verfassten

Stellungnahmen

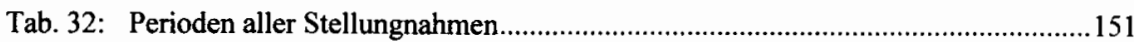

Tab. 33: Anzahl der Konstellationen bei den Erststellungnahmen.....................................154

Tab. 34: Anzahl der Konstellationen ohne Veränderung im Prozess ...................................154

Tab. 35: Veränderungen der Konstellationen im Zeitablauf .............................................. 154

Tab. 36: Anzahl und Anteil der Koalitionen und Konstellationen im Zeitablauf ................156

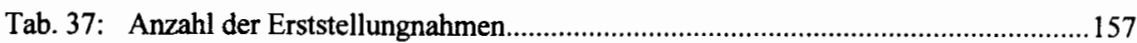

Tab. 38: Reihenfolge der Zusammenschlüsse zwischen den Interessengruppen .................158

Tab. 39: Reihenfolge des Eintrittes in die Konstellationen bei gegensätzlichen Meinungen. 158

Tab. 40: Anzahl der von Interessengruppen und/oder dem Regulierer thematisierten Rechnungslegungsprobleme

Tab. 41: Konstellationen der Interessengruppen in Bezug auf den Regulierer ...................162

Tab. 42: Meinungsänderung des Regulierers .............................................................. 163

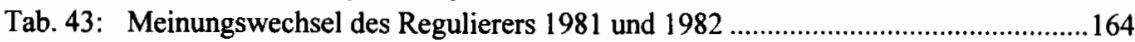

Tab. 44: Meinungswechsel des Regulierers 1985,1* ....................................................... 165

Tab. 45: Meinungswechsel des Regulierers 1985,2 und 1985,3 ....................................... 165

Tab. 46: Meinungswechsel des Regulierers 1985,4 ......................................................... 166

Tab. 47: Kodierung des Periodenerfolgs der drei ausgewählten Stellungnahmen der KRW, Vorentwurf 1980.

Tab. 48: Kodierung des Periodenerfolgs der drei ausgewählten Stellungnahmen der KRW, Referentenentwurf 1981

Tab. 49: Kodierung des Periodenerfolgs der drei ausgewählten Stellungnahmen der KRW, Unterausschuss-Entwurf 1985,2

Tab. 50: Kodierung des Periodenerfolgs der drei ausgewählten Stellungnahmen der KRW, Unterausschuss-Entwurf 1985,3

Tab. 51: Anzahl und Erfolg der Koalitionen ohne Gegenstimmen im Entwurf 1982

Tab. 52: Anzahl und Erfolg der Koalitionen mit Gegenstimmen im Entwurf 1982 ........... 174

Tab. 53: Anzahl und Erfolg der Interessengruppen in Koalitionen ohne Gegenstimmen im Regierungsentwurf 1982

Tab. 54: Anzahl und Erfolg der Interessengruppen in Koalitionen mit und ohne Gegenstimmen im Entwurf 1982 .

Tab. 55: Anzahl der ÄE und BE sowie der ÄM und BM der KRW im Zeitablauf und deren Anteil am Gesamterfolg und -misserfolg

Tab. 56: Anzahl der ÄE und BE sowie der ÄM und BM des IDW im Zeitablauf und deren Anteil am Gesamterfolg und -misserfolg 
Tab. 57: Anzahl der ÄE und BE sowie der ÄM und BM der GEFIU im

Zeitablauf und deren Anteil am Gesamterfolg und -misserfolg. 177

Tab. 58: Anteil der ÄE und BE sowie der ÄM und BM der SPVDW im

Zeitablauf und deren Anteil am Gesamterfolg und -misserfolg. 178

Tab. 59: Regressionen zur Vorhersage des Erfolges der Interessengruppen 


\section{Verzeichnis der verwendeten Symbole}

$\ddot{\mathrm{AE}}$

$\ddot{A M}$

$\mathrm{BE}$

$\mathrm{BM}$

$\mathrm{C}$

D

E

EQ

G

GI

I

IW

$\mathrm{K}$

$\mathrm{KV}$

$\mathrm{M}$

MI

MW

OI

$P$

$\mathrm{R}$

Reg

RP

SN

S

$\mathrm{U}$

V1Z1

WA
Änderungserfolg

Änderungsmisserfolg

Beibehaltungserfolg

Beibehaltungsmisserfolg

Kosten

persönliche Befriedigung

Eigenmeinung des Regulierers

Erfolgsquote

Gesellschaft für Finanzwirtschaft in der Unternehmensführung

e.V.

Meinungswechsel gegen Interessengruppen

Institut der Wirtschaftsprüfer

Interessengruppenwechsel

Kommission Rechnungswesen

Keine Veränderung

Meinung des Regulierers

Meinungswechsel zu Interessengruppen

Meinungswechsel des Regulierers

Meinungswechsel ohne Interessengruppeneinfluss

Wahrscheinlichkeit

Nutzen der Beteiligung

Regulierer

Rechnungslegungsproblem

(Anzahl der) Stellungnahme(n)

Spitzenverbände der deutschen Wirtschaft

Nutzen der Wahlalternative

Meinungswechsel von einer zur anderen Interessengruppe

Wiederaufnahme 


\section{Abkürzungsverzeichnis}

Abb.

Abl.

Abs.

ADHGB

ADS

AE

a.F.

AktG

APB

Art.

ASB

Aufl.

Az.

BankBiRiLiG

BB

$\mathrm{Bd}$.

BDA

BDB

BDI

Bewertungsgrunds.

BFH

BFuP

BGBI.

BilReG

BiRiLiG

bspw.

BStB1.

BT

bzw.

CDU

Co.

$\mathrm{CSU}$

D.C.

d.h.

DB

DBW

DIHT

DMBiG

DRSC

DStR
Abbildung

Amtsblatt

Absatz

Allgemeines Deutsches Handelsgesetzbuch

Adler/Düring/Schmaltz

Ausschussentwurf

alte Fassung

Aktiengesetz

Accounting Principles Board

Artikel

Accounting Standards Board

Auflage

Aktenzeichen

Bankbilanzrichtlinie-Gesetz

Betriebs-Berater

Band

Bundesvereinigung der Deutschen Arbeitgeberverbände

Bundesverband Deutscher Banken

Bundesverband der Deutschen Industrie

Bewertungsgrundsätze

Bundesfinanzhof

Betriebswirtschaftliche Forschung und Praxis

Bundesgesetzblatt

Bilanzrechtsreformgesetz

Bilanzrichtlinien-Gesetz

beispielsweise

Bundessteuerblatt

Bundestag

beziehungsweise

Christlich Demokratische Union

Compagnie

Christlich-Soziale Union

District of Columbia

das heißt

Der Betrieb

Die Betriebswirtschaft

Deutscher Industrie- und Handelstag

Gesetz zur Änderung des D-Markbilanzgesetzes und anderer handelsrechtlicher Bestimmungen

Deutsches Rechnungslegungs Standards Committee

Deutsches Steuerrecht 
e.V.

ED

EG

EGHGB

EStG

et al.

EU

EuGH

f.

FASB

FDP

ff.

FIFO

finanz.

FN

GAAP

GEFIU

ges

GeschO BT

GeschO BM

GG

ggf.

$\mathrm{GmbH}$

GmbHG

GmbHR

GoB

grunds.

GuV

HGB

HIFO

HRefG

Hrsg.

hrsg.

IAS

IASB

IASC

IDW

IFRS

insb.

Jg.

KapAEG eingetragener Verein

Exposure Draft

Europäische Gemeinschaften

Einführungsgesetz zum $\mathrm{HGB}$

Einkommensteuergesetz

et alii

Europäische Union

Europäischer Gerichtshof

folgende

Financial Accounting Standards Board

Freie Demokratische Partei

fortfolgende

First-in-First-Out

finanzielle

Fußnote

Generally Accepted Accounting Principles

Gesellschaft für Finanzwirtschaft in der Unternehmensführung

e.V.

gesamt

Geschäftsordnung des Deutschen Bundestages

Gemeinsame Geschäftsordnung der Bundesministerien

Grundgesetz

gegebenenfalls

Gesellschaft mit beschränkter Haftung

GmbH-Gesetz

GmbH-Rundschau

Grundsätze ordnungsmäßiger Buchführung/Bilanzierung

grundsätzlich

Gewinn- und Verlustrechnung

Handelsgesetzbuch

Highest-in-First-Out

Handelsrechtsreformgesetz

Herausgeber

herausgegeben

International Accounting Standard

International Accounting Standards Board

International Accounting Standards Committee

Institut der Wirtschaftsprüfer

International Financial Reporting Standards

insbesondere

Jahrgang

Kapitalaufnahmeerleichterungsgesetz 


\section{Kap-CoRiLiG}

$\mathrm{KG}$

KonTraG

KoR

KRW

LIFO

m.w.N.

No.

$\mathrm{Nr}$.

n.s.

PublG

RAP

$\mathrm{RE}$

RegE

Rückst.

$\mathrm{Rz}$.

$\mathrm{S}$.

SEC

SFAS

sog.

Sp.

SPD

SpVdW

StuW

Tab.

TransPuG

u.a.

U.K.

US, U.S.

USA, U.S.A.

US-GAAP

$\mathrm{VE}$

Verb.

Vereinfachungsverf.

Verpfl.

VersRiLiG

vgl.

VO-Ermächtigungen

Vol.

WPg

WPK

z.B.
Kapitalgesellschaften- und Co-Richtlinie-Gesetz

Kommanditgesellschaft

Gesetz zur Kontrolle und Transparenz im Unternehmensbereich

Zeitschrift für kapitalmarktorientierte Rechnungslegung

Kommission Rechnungswesen im Verband der Hochschullehrer

Last-in-First-Out

mit weiteren Nachweisen

number

Nummer

nicht signifikant

Publizitätsgesetz

Rechnungsabgrenzungsposten

Referentenentwurf

Regierungsentwurf

Rückstellungen

Randziffer

Seite

Securities and Exchange Commission

Statement of Financial Accounting Standards

so genannte

Spalte

Sozialdemokratische Partei Deutschlands

Spitzenverbände der deutschen Wirtschaft

Steuer und Wirtschaft

Tabelle

Transparenz- und Publizitätsgesetz

unter anderem

United Kingdom

United States

United States of America

US-Generally Accepted Accounting Principles

Vorentwurf

Verbindlichkeiten

Vereinfachungsverfahren

Verpflichtung

Versicherungsbilanzrichtlinie-Gesetz

vergleiche

Verordnungsermächtigungen

Volume

Die Wirtschaftsprüfung

Wirtschaftsprüferkammer

zum Beispiel 
ZfB

$\mathrm{ZfbF}$

Zf.
Zeitschrift für Betriebswirtschaft

Zeitschrift für betriebswirtschaftliche Forschung Ziffer 